\title{
Design of Real-Time Monitoring of Infant Physical Condition in Sports Training Based on Embedded Theory
}

\author{
Biao He $\left.{ }^{1}\right)^{1}$ and Yuwen Wan ${ }^{2}$ \\ ${ }^{1}$ College of Sports Science, Hengyang Normal University, Hengyang, 421010 Hunan, China \\ ${ }^{2}$ School of Education and Sports, Jiangxi University of Applied Science, Nanchang, 330100 Jiangxi, China \\ Correspondence should be addressed to Biao He; hb3379@hynu.edu.cn
}

Received 25 October 2021; Revised 6 December 2021; Accepted 21 December 2021; Published 20 January 2022

Academic Editor: Haibin Lv

Copyright (C) 2022 Biao He and Yuwen Wan. This is an open access article distributed under the Creative Commons Attribution License, which permits unrestricted use, distribution, and reproduction in any medium, provided the original work is properly cited.

\begin{abstract}
Children at this age are a critical period of physical and mental development in a person's life. Correctly understanding and grasping the characteristics and laws of physical and mental development of children at this stage and developing targeted sports and training are conducive to the physical development and growth of children. The first is the concrete image of the cognitive activity; the second is the unintentional nature of mental activities and behaviors; the third is the initial tendency of personality in order to monitor the physical condition of children in sports training in real time and to improve the management and data analysis capabilities of the physical condition of children in sports training; a set of real-time monitoring system for the physical condition of children in sports training is designed to help develop and improve the intelligence of children's sports training management. This article mainly introduces the design of a real-time monitoring system for children's physical condition in sports training based on an embedded system and is aimed at providing some references for the real-time monitoring of the physical condition of young children in sports training. This paper proposes a design method of a real-time monitoring system for children's physical fitness in sports training based on embedded systems, utilizing the professionalism and real time and tailored the ability, reliability, and low power consumption of the embedded system to design a real-time monitoring system for the physical condition of young children in sports training; reuse includes the document retrieval, questionnaire survey, mathematical statistics, logical analysis, and real-time monitoring related algorithms, such as a real-time monitoring system based on the Internet of Things system, which is used for children's physical fitness in sports training. Real-time monitoring system designed research experiment. The results show that the embedded real-time monitoring system designed in this paper has an average accuracy rate of $93.33 \%$ for children's physical conditions, and the accuracy is strong, and it can be applied to the monitoring of children's physical condition; it can promote the good development of children's body and physique and improve the intelligent management ability of children's sports training.
\end{abstract}

\section{Introduction}

With the rapid development of microprocessors, the performance of current microprocessors is getting higher and higher; a microprocessor is a central processing unit composed of one or a few large-scale integrated circuits. Compared with the traditional central processing unit, it has the advantages of small size, light weight, and easy modularization. A new research topic that combines embedded systems with human body fitness parameter monitoring has emerged. With its fast data processing capabilities and good network performance, it has been well developed in many fields.

Embedded systems take application as the core microelectronics technology and control technology, emphasizing the collaboration and integration of materials and software; software and equipment can adapt to system's operation, reliability, cost, and size; a performance dedicated computer system with strict requirements on the consumption and application environment. In view of the current weak initiative of ordinary video monitoring systems, the strong customization of professional intelligent monitoring systems, 
and the many advantages of embedded platforms, an embedded real-time monitoring system for the physical condition of children in sports is proposed, which can be used when children are playing sports. Real-time monitoring of children's moving human bodies in the monitoring scene and automatically saves the monitored physical fitness information to the designated path of the system.

Zeng et al. believe that today's traditional stand-alone embedded systems are limited in terms of functionality, flexibility, and scalability, because of its limited resources, limited processing power, and high hardware dependence. The role of the fog computing platform is to push cloud services to the edge of the network. This is a way to support and enhance traditional outlook solutions. Integrated system resource management has always been the key to system performance. Zeng et al. studied the software built-in system supported by the fog computer, in which the working image is located on the storage server, and the computer can be run on the built-in device or computer server; we designed an efficient task scheduling resource management strategy to minimize the task completion time improving user experience. Zeng et al. studied three issues: how to balance the workload between client devices and computer servers; how to place working image storage servers on the servers; and how to balance I/O interruption requests between storage servers. This research process is relatively cumbersome, difficult to be applied in reality [1]. Back et al. introduced the development of low-cost embedded energy management systems for industrial environments. The main goal is to use digital signal processing (DSP) technology to monitor, collect, and analyze electrical parameters. For data analysis, Back et al. have created two applications: one uses Matlab ${ }^{\circledR}$ software; the other is derived from the first application and runs on an embedded device. Both of these applications use discrete wavelet transform as the main technique for interference detection. This research is rather one-sided and not practical [2]. Huang developed a new intelligent neuro-fuzzy surface roughness monitoring system in his research. The purpose of this model is to improve the accuracy of surface roughness prediction and solve the shortcomings of neural networks and fuzzy logic. They use statistical techniques to analyze the accuracy between these systems. However, the system is still in the preliminary investigation stage and needs further investigation [3].

The innovations of this article are: (1) proposed realtime monitoring algorithms for the design of a real-time monitoring system for children's physical condition; (2) carried out the hardware design of a real-time monitoring system for children's physical conditions; (3) designed a real-time monitoring system for children's physical condition monitoring system-related software.

\section{Design Method of Real-Time Monitoring System for Infant Physical Condition in Sports Training Based on Embedded System}

2.1. Research Method. This article uses the monitoring technology under the Internet of Things environment to collect data on the actual information of children's physical exercise. The following is the calculation and processing method of the influencing factors that may appear in the monitoring process:

2.1.1. Document Retrieval Method. According to the needs of this research and the characteristics of the research content, through CNKI, AOL, Springer Link, government websites, and other channels, combined with network query, database access, book borrowing, and resource purchase, etc., a wide range of embedded monitoring systems, children's physique monitoring, and other related literature materials are summarized, sorted, and analyzed to provide theoretical references for the research of this topic [4].

\subsubsection{Questionnaire Survey Method}

(1) There are two kinds of questionnaires designed in this study, namely, teacher's questionnaire for monitoring the physical condition of children in sports training and the questionnaire for experts related to the monitoring of physical condition during human sports training. The teacher questionnaire is designed based on preliminary surveys and a large number of relevant literatures. According to the needs of this research, it is designed to consult relevant experts and relevant leaders and teachers involved in the monitoring of children's physical status. The purpose is to understand the current status of children's physical status, and there are problems in physical fitness monitoring; the expert questionnaire is designed on the basis of previous interviews with relevant school leaders and physical education teachers engaged in the monitoring of children's physical fitness $[5,6]$

(2) The validity test of the questionnaire is an indispensable link in the research and a prerequisite for the authenticity, validity, and credibility of the questionnaire data. The opinions of relevant experts and professors were extensively solicited during the design of the questionnaire, and the questionnaire effectiveness was formulated by the content and structure of the questionnaire involved in this research, degree evaluation table [7]

2.1.3. Mathematical Statistics. Use software such as EXCEL and SPSS to process and analyze the data obtained in this study, and draw various statistical charts, etc., to provide reliable data support for the study [8].

2.1.4. Logic Analysis Method. Use logic principles such as induction, deduction, analogy, and synthesis to analyze the collected data and combine the theoretical knowledge you have learned to make the research systematized and theoretical, conduct comprehensive analysis and discussion, and get more scientific research conclusions and recommendations [9].

\subsection{Real-Time Monitoring-Related Algorithms}

2.2.1. Error Compensation. Error compensation refers to the calculation of nonlinear errors to obtain their accurate errors and compensate them. The formula is: 


$$
\delta_{1}=\left(\frac{\Delta 1_{\max }}{\Sigma}\right) \times 100 \% F \cdot S .
$$

$\Sigma$ represents the theoretical full-scale output value, and the variable $\Delta 1_{\max }$ represents the maximum deviation $[10,11]$.

$$
y=k x+b
$$

The residual error between the itch calibration data $y_{i}$ and the corresponding value of the fitted straight line is

$$
\Delta i=y_{i}-(k x+b)
$$

according to the principle of least squares method, and set to zero to obtain $k$ and by $[12,13]$. The announcement is as follows:

$$
\begin{array}{r}
k=\frac{10 \sum x_{i} y_{i}-\sum x_{i} \cdot \sum y_{i}}{10 \sum x_{i}^{2}-\left(\sum x_{i}\right)^{2}}, \\
b=\frac{\sum x_{i}^{2} \cdot \sum y_{i}-\sum x_{i} \cdot \sum x_{i} y_{i}}{10 \sum x_{i}^{2}-\left(\sum x_{i}\right)^{2}} .
\end{array}
$$

2.2.2. Filtering Algorithm. There are many filtering methods for weak signal detection algorithms. The algorithm used to monitor the stability of baby's physical condition optimizes the sampling frequency error analysis results $[14,15]$.

Differential signals with strong anti-interference capability are used for signal acquisition [16, 17]. The basic formula of FIR is:

$$
\begin{gathered}
A(i)=\sum_{i-1}^{a-1} b(j) \cdot B(i-1), \quad j=0,1,2, \cdots, M, \\
S(z)=\frac{1}{P} \sum_{i-1}^{P-1} K_{i}(d) z^{-i}, \\
O\left(R^{j w}\right)=\frac{1}{P} \sum_{i-1}^{T-1} K_{i}(d) R^{-j w n}, \\
A(r)=\frac{(b(r)+b(r-1)+b(r-2)+b(r-3))}{4} .
\end{gathered}
$$

where the transfer function can be expressed as:

$$
h(z)=\frac{1+z^{-1}+z^{-2}+z^{-3}}{4} .
$$

Substituting $\mathrm{z}=e^{j \theta n}$, the frequency characteristic formula is:

$$
h\left(e^{j \theta n}\right)=\frac{1+e^{-j \theta n}+e^{-2 j \theta n}+e^{-3 j \theta n}}{4},
$$

TABle 1: Part of the technical process of this method.

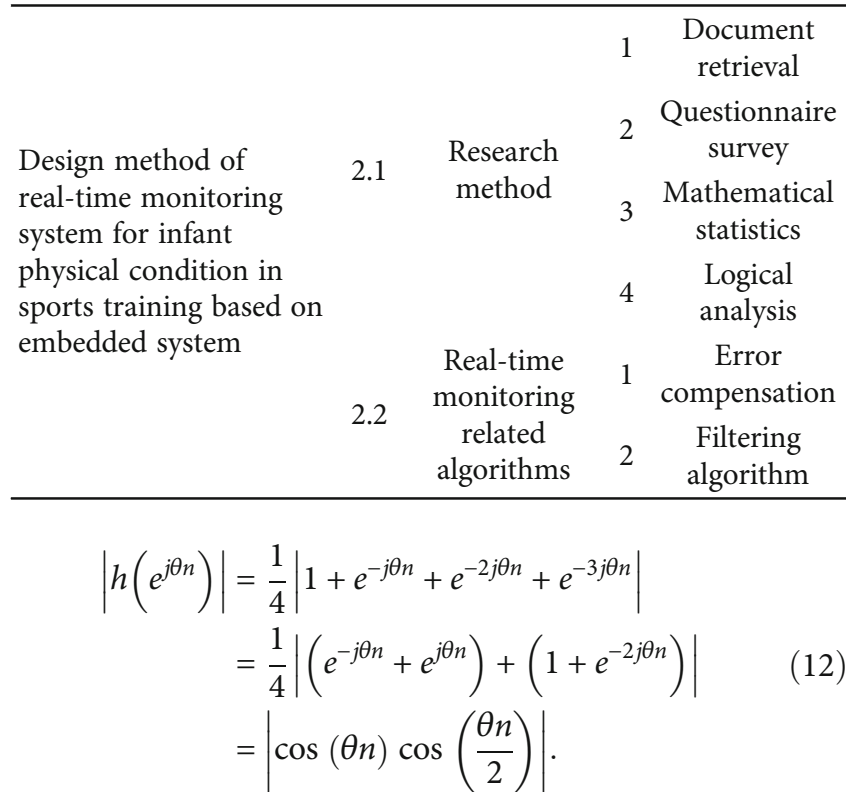

As shown below:

$$
\begin{gathered}
\phi=\sum_{i=0}^{M} e^{2}(i)=\sum_{i=0}^{M} d^{2}(i)-2 \sum_{k=0}^{N-1} K(d) r_{d x}(d) \\
+\sum_{k=0}^{N-1} \sum_{j=0}^{N-1} K(d) K(i) r_{x x}(d-k) \\
\frac{\partial \phi}{\partial w(k)}=0, \quad 0 \leq K \leq N-1 .
\end{gathered}
$$

Find the best fitness weight coefficient $w(k)$, and get

$$
\begin{aligned}
\phi= & \sum_{i=0}^{M} e^{2}(i)=\sum_{i=0}^{M} d^{2}(i)-2 \sum_{k=0}^{N-1} K(d) r_{d x}(d) \\
& +\sum_{k=0}^{N-1} \sum_{j=0}^{N-1} K(d) K(i) r_{x x}(d-k) .
\end{aligned}
$$

The specific process is shown in Table 1.

\section{Design of a Real-Time Monitoring System for Infants' Physical Fitness in Sports Training Based on Embedded System}

\subsection{System Hardware Design}

3.1.1. Overview of System Hardware Design. The basic layer of the integrated system is the central processing unit, which contains the digital unit and the controller unit. Based on the processor, combined with memory module, power module, recovery module, etc., a so-called minimal system is formed. The embedded system level and hardware structure are shown in Figure 1.

The hardware circuit of the embedded system designed in this article is mainly divided into eight parts, MCU circuit, 


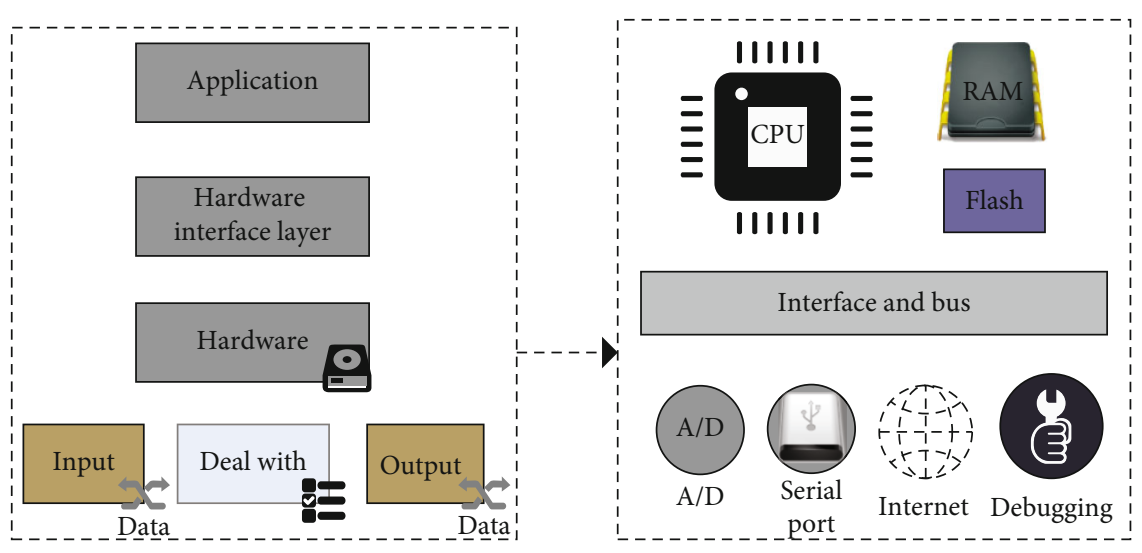

FIgURE 1: Embedded system level and hardware structure.

Bluetooth circuit, ECG acquisition circuit, body temperature measurement circuit, pressure sensor, power supply circuit, FLASH storage module, MCU received ECG acquisition module, and body temperature through USART and I2C bus, respectively, Real-time monitoring data sent by the sensor. The pressure sensor and the MCU realize data transmission through the $\mathrm{A} / \mathrm{D}$ conversion circuit. The MCU analyzes the data and stores it in the FLASH memory. Flash memory is a nonvolatile memory; its storage characteristics are equivalent to a hard disk and can retain data for a long time without current supply [18]. When the MCU receives the command sent by Android to retrieve the measurement data, the corresponding data will be processed. It is sent through USART and transmitted to Android smart terminal through Bluetooth module $[19,20]$. Therefore, the physical condition data can be quickly updated and displayed in real time, and it is easy to carry out and has high practicality.

3.1.2. System MCU Peripheral Circuit Design. In the system, the MCU is mainly responsible for data processing and corresponding transparent transmission. MCU is a single-chip microcontrol computer based on the central processing unit. There are many types of data transmitted by the system, and the algorithms involved are also more complicated. There are many types of data transmitted by the system, and the algorithms involved are more complex. Therefore, the performance of the MCU processor is higher. After comparing multiple microcontroller processors, the final makes sure to use STM32F103RCT6 microcontroller as the main control processor of the hardware system terminal. STM32F103RCT6 is a 32-bit embedded high-speed processor under the Cortex$\mathrm{M} 3$ architecture. It runs at $72 \mathrm{MHZ}$ and integrates $512 \mathrm{~KB}$ bytes of high-speed memory and $48 \mathrm{~KB}$ bytes of RAM space. Peripheral equipment connection resources are more abundant, up to 50 IOs, 8 timers, and 12-bit $\mathrm{AD}$ and DA converters [21]. The current can be reduced to $2 \mathrm{uA}$ in standby mode. It contains multiple USART universal serial communication interfaces, providing a more convenient means of transmission for system development. It supports the use of JTAG emulation debugger, which makes it more convenient to use the online debugging function. It also contains multiple serial peripheral interfaces (SPI) and other bus protocol interfaces [22].

3.1.3. System Power Circuit Design. To make the portability of the system more prominent, it is convenient to carry around. The system uses 18650 lithium-ion batteries for power supply. The $2000 \mathrm{mAh} 18650$ battery can support the system to work continuously for up to 100 hours. The control part of the power supply circuit is realized by SN8P2711A microcontroller, which is a RISE-like high-performance, low-power MCU, which is widely used and low in price. The control circuit of the system mainly completes the functions of collecting voltage, controlling, the working status of charging and discharging, and power indicator. The charging management circuit adopts TC4056. TC4056 is a linear charging chip specially designed for lithium ion or lithium polymer batteries. The integrated power supply circuit in the chip can realize constant current or constant voltage charging of external batteries, respectively [23]. The charging current can be changed by adjusting the resistance of the external resistor. The maximum charging current can reach $1 \mathrm{~A}$, which is fully applicable to USB power supply and adapter power supply.

3.1.4. Microprocessor Chip. The LPC2132 microprocessor is a 32/16-bit ARM7TDMI-S CPU, which is very powerful and can support real-time simulation and embedded tracking. It has $32 \mathrm{~KB}, 64 \mathrm{~KB}, 128 \mathrm{~KB}, 256 \mathrm{~KB}$, and $512 \mathrm{~KB}$ single-chip embedded high-speed flash memory. It has a 128-bit width memory interface and a unique acceleration structure, which enables the LPC2132 microprocessor to make 32-bit code run perfectly at the maximum clock rate. For core code applications, 16-bit thumb mode can be used. In this case, we can significantly reduce the performance loss of the code, which may exceed $30 \%$. The LPC 2132 microprocessor is very suitable for communication gateways, protocol converters, soft modems, voice recognition, and low-end imaging. It also provides powerful data processing capabilities and a large buffer space. It has multiple 32-bit timers, single or dual 10-bit 8-channel ADC(S), 10-bit DAC, PWM channels, and 47 GPIOs, as well as up to 9 edge or level-triggered external interrupt pins, which makes LPC2132 microprocessors widely used in industrial control and medical systems. 


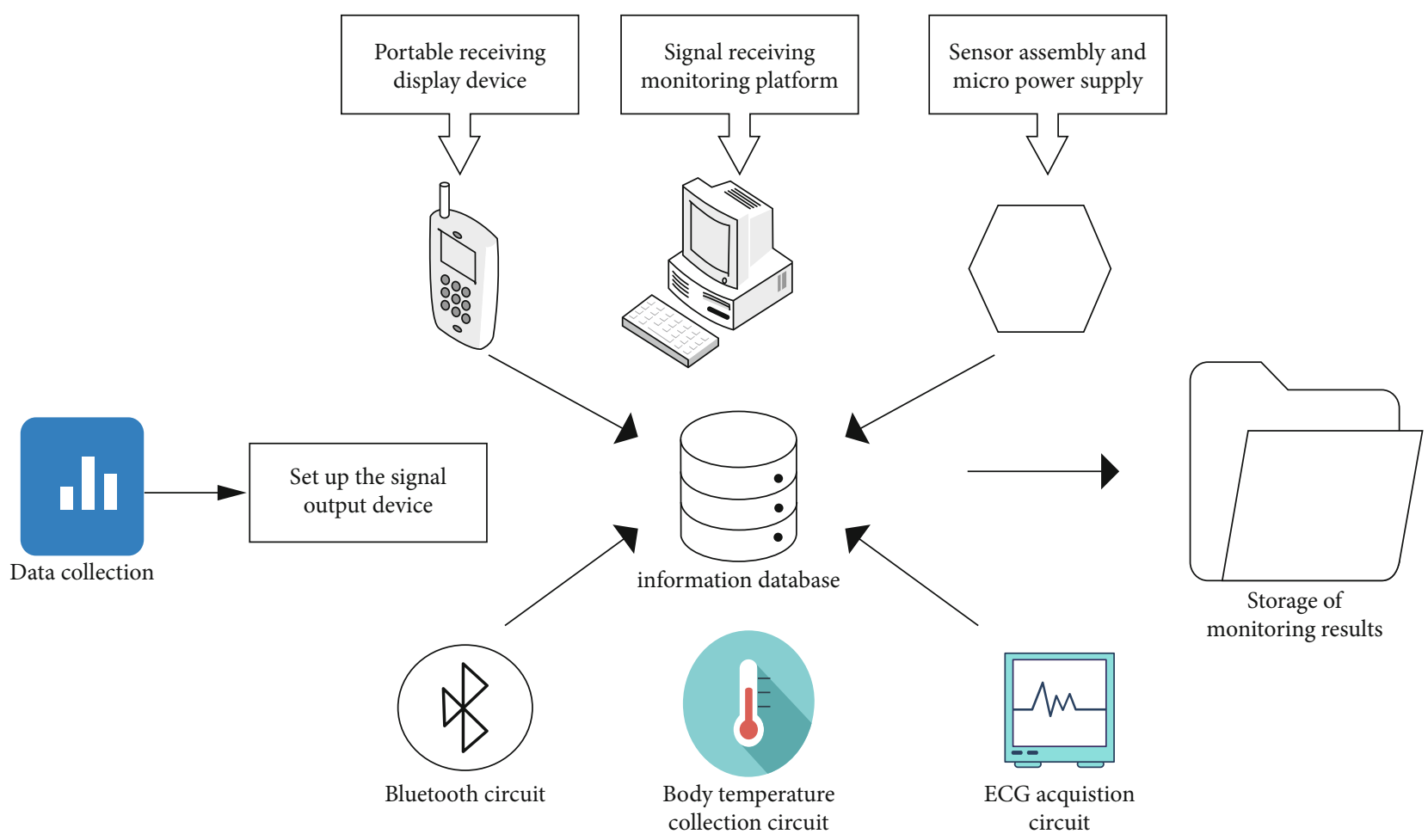

FIGURE 2: System overall design framework diagram.

TABle 2: Some steps of this experiment.

\begin{tabular}{lccc}
\hline & Design of a real-time monitoring system for infants' physical fitness in sports training based on embedded system \\
\hline 3.1 & System hardware design & 3.2 & System software design \\
1 & System hardware design overview & 1 & PC operating system selection \\
2 & System MCU peripheral circuit design & 2 & Development platform construction \\
3 & System power circuit design & 3 & Data storage design \\
4 & Microprocessor chip & 4 & Software design of lower computer \\
\hline
\end{tabular}

TABLE 3: Evaluation of children's physical fitness levels.

\begin{tabular}{lccc}
\hline Category & Boys & Girls & Total \\
\hline Excellent & $7.62 \%$ & $8.41 \%$ & $8.02 \%$ \\
Good & $24.75 \%$ & $31.06 \%$ & $27.91 \%$ \\
Eligible & $58.49 \%$ & $56.21 \%$ & $57.35 \%$ \\
Ineligible & $9.14 \%$ & $4.32 \%$ & $6.73 \%$ \\
Pass rate & $90.86 \%$ & $95.68 \%$ & $93.27 \%$ \\
\hline
\end{tabular}

\subsection{System Software Design}

3.2.1. PC Operating System Selection. The upper computer operating system is the link between the hardware equipment and the software system. It directly responds to the operation of the software program to manage and control the hardware equipment. With the development of the Internet and computers, common operating systems include Windows and Linux; for embedded devices, there are also embedded Linux, WinCE, and VxWorks; Vxworks is generally used in military and aerospace equipment, so the information is relatively confidential, and the cost of learning and development is also very high; Linux is more general and popular and has a wide range of applications; WinCE is a Microsoft module, and the stability is not as good as Linux, but the graphical interface is very dazzling, so it is often used in consumer electronics fields such as smart phones [24]. In mobile-embedded devices, especially consumer electronics such as smart phones and tablet computers, Symbian, Android, iOS, Windows, and Phone are mainly used. Mature embedded systems are often used in industrial production, and good interactive interfaces and user experience make mobile-embedded operating systems more suitable for use in mobile electronic devices.

3.2.2. Development Platform Construction. Android's development language is Java, so you must first download and install the JDK (Java Development Kit). The minimum requirement 


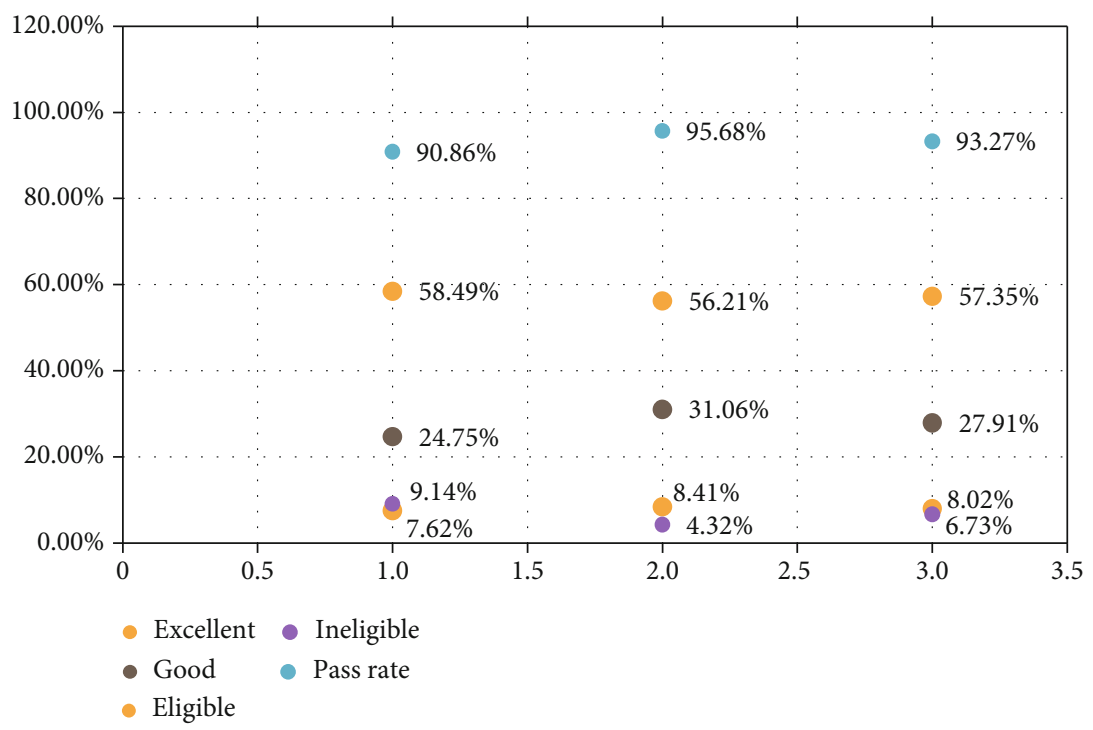

FIGURE 3: Young children's physical fitness rating.

TABLE 4: Overall compliance rate of children of different ages.

\begin{tabular}{lcccc}
\hline Sex & 3 years old & 4 years old & 5 years old & 6 years old \\
\hline Boys & $86.26 \%$ & $89.45 \%$ & $91.63 \%$ & $90.18 \%$ \\
Girls & $88.37 \%$ & $90.58 \%$ & $89.74 \%$ & $88.29 \%$ \\
Total & $87.31 \%$ & $90.02 \%$ & $90.69 \%$ & $89.24 \%$ \\
\hline
\end{tabular}

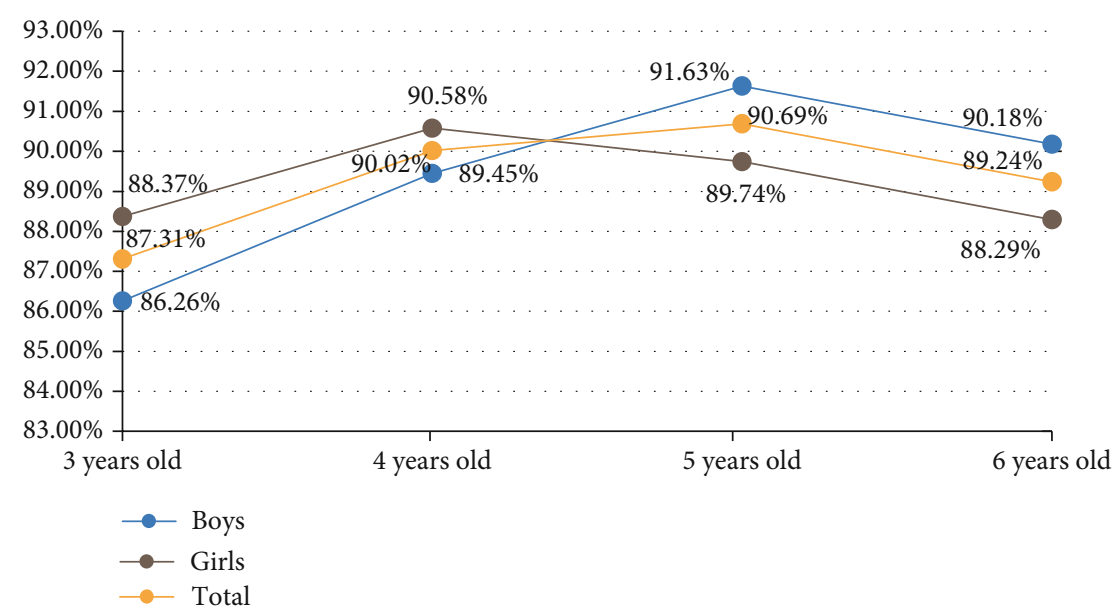

FIgURE 4: Overall compliance rate of children of different ages.

for Android application development is Java SE6, and Java SE7 is currently commonly used. After the installation is complete, the environment variables must be configured, and the samples can be sent to compile and run the written program.

3.2.3. Data Storage Design. SQLite database stores data. SQLite is a pinch-level-embedded database engine. It supports SQL language and has very good performance with very little memory.
3.2.4. Software Design of Lower Computer. The lower-level function is relatively simple, but the data is collected directly, so the focus is on the accuracy and immediacy of the collected data. Since the lower computer itself is an independent monitoring device, the stand-alone mode (away from the control of the upper computer) is added to achieve product independence. The serial port display is selected on the display, and this function is realized through the serial port 1 of the single-chip microcomputer. By understanding 
TABLE 5: Questionnaire survey reliability results.

\begin{tabular}{lccccc}
\hline Evaluation standard & Very reasonable & Reasonable & Basically reasonable & Not reasonable & Not reasonable at all \\
\hline Structure & 7 & 8 & 4 & 1 & 1 \\
Content & 8 & 6 & 4 & 3 & 0 \\
Logic & 9 & 8 & 4 & 0 & 0 \\
Design & 6 & 9 & 4 & 1 \\
\hline
\end{tabular}

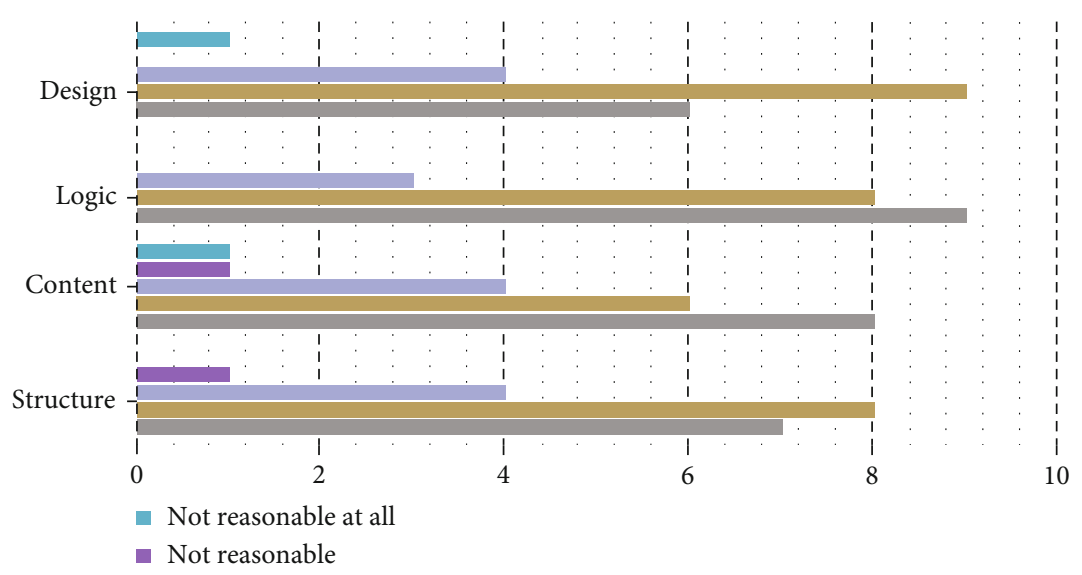

FIGURE 5: Questionnaire validity.

the characteristics of the display screen, $9600 \mathrm{bps}$ is used for communication, and the serial port format is 8 data bits, 1 stop bit, and no parity bit. Set the parameters of the MCU serial port 1 according to the serial communication requirements of the display screen.

3.2.5. Monitoring Process Design. The software design in stand-alone mode considers the following two situations. One is the test mode, which realizes the independent test process, including the collection of information of the tested person (here only manual entry is used), time control, data collection, and result storage, and the addition of the function of viewing and deleting results; the other is practice mode, considering the actual application scenarios and equipment testing, and practice mode, that is, there is no need to enter information or save data. The real-time monitoring design process is shown in Figure 2.

In the online mode, the collection of results is the same as the stand-alone mode. The test time is also controlled by the lower computer to ensure the accuracy of the time, and the start signal of the time is controlled by the upper computer to achieve fairness of the test. Because the host computer has already saved the data, the local save design is no longer done. Wireless communication also uses serial communication. Serial communication, as one of the computer communication methods, mainly plays the role of data transmission between the host and peripherals and the host. Serial communication has the characteristics of fewer transmission lines and low cost. Because the importance of wireless data is higher than that of serial screens, serial 0 is used. Serial port 0 is available during power-on initialization, but the serial port is closed after the stand-alone mode is selected. And in the online mode, the control is done by the host, so the operation of responding to the serial port screen is not performed, and the serial port 1 only sends but not receives $[25,26]$.

The detailed process of embedded system real-time monitoring is shown in Table 2.

\section{Design and Analysis of a Real-Time Monitoring System for Children's Physical Fitness in Sports Training Based on Embedded System}

\subsection{Questionnaire Analysis}

(1) By conducting a questionnaire survey of kindergarten teachers, sorting out, and analyzing the results of the questionnaire, we can get a rough assessment of the physical fitness level of the children, as shown in Table 3 and Figure 3

From the chart, it can be seen that the total score of excellent male and female children is relatively small, and most of them are concentrated in passing standards. The good rate of female children is higher than that of men; the passing rate of female children is also higher than that of men; the failing rate of male children is higher than that of females. The overall excellent rate of male and female children is $8.02 \%$; the pass rate is $93.27 \%$.

(2) By conducting a questionnaire survey on kindergarten teachers, sorting out and analyzing the results of the questionnaire, we can get a rough rate of reaching the physical fitness standard of children. The specific situation is shown in Table 4 and Figure 4 
TABLE 6: Questionnaire reliability test results.

\begin{tabular}{lcccc}
\hline & Questionnaire structure & Questionnaire content & Questionnaire logic & Questionnaire design \\
\hline Cronbach's alpha $(\alpha)$ & 0.903 & 0.896 & 0.917 & 0.921 \\
\hline
\end{tabular}

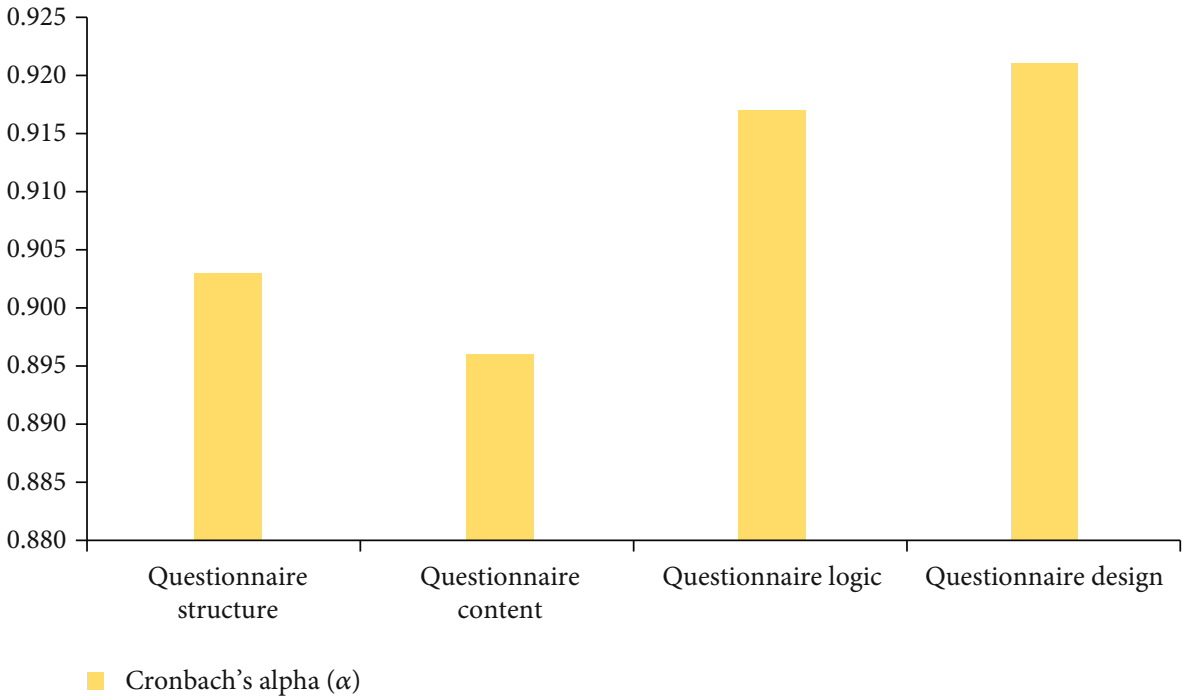

Figure 6: Questionnaire reliability test results.

TABLE 7: Algorithm processing speed comparison.

\begin{tabular}{lccc}
\hline Video clip & $\begin{array}{c}\text { Include } \\
\text { frames }\end{array}$ & $\begin{array}{c}\text { Fir processing } \\
\text { time }(\mathrm{s})\end{array}$ & $\begin{array}{c}\text { Improved } \\
\text { processing time }(\mathrm{s})\end{array}$ \\
\hline Bootstrap & 2971 & 159.15 & 136.28 \\
LightSwitch & 2546 & 156.37 & 133.59 \\
MovedObject & 1973 & 49.26 & 36.61 \\
Time of day & 5428 & 253.42 & 214.48 \\
Average & 3230 & 154.55 & 130.24 \\
\hline
\end{tabular}

It can be seen that the overall compliance rate of children in different age groups is at a relatively high level, exceeding $85 \%$. The compliance rate of male and female children first increased and then decreased with age, and the compliance rate of male and female children reached the maximum at 5 years old. Before the age of 5 , the compliance rates of female children in other age groups were higher than those of male children; after the age of 5, the compliance rates of female children in other age groups were lower than that of male children.

(3) Through 20 experts, make a five-level validity test of "very reasonable, reasonable, general, unreasonable, and very unreasonable" and some suggestions for revision of the questionnaire, summarize the questionnaire, exclude invalid factors, and guarantee this article the validity of the questionnaire. The validity of the statistical questionnaire is shown in Table 5 and Figure 5
As shown in the data in the table, after statistical analysis of the evaluation results and the validity, evaluation results of the three questionnaires are all reasonable and above, which can prove that experts generally believe that the overall design of the questionnaire is reasonable and can more objectively reflect the requirements. The results sought that the structure of the questionnaire is relatively reasonable, and the language expression is relatively clear, which can meet the needs of scientific research, and the questionnaire survey can be implemented.

(4) This article mainly uses the test-retest reliability analysis method in the questionnaire reliability testing process, using the commonly used Cronbach's Alpha reliability coefficient analysis in SPSS. Cronbach's analysis is mainly used to evaluate the consistency of continuous variables and ordinal categorical variables. When the reliability coefficient is less than 0.5 , the questionnaire is credible; when the reliability coefficient is between 0.5 and 0.8 , the reliability of the questionnaire is acceptable; when the reliability coefficient of the questionnaire is greater than or equal to 0.8 , the reliability of the questionnaire is very good. Use SPSS 25.0 software to further analyze and questionnaire survey. The reliability results of the questionnaire are shown in Table 6 and Figure 6

Calculating from the data in the table, the average value of the correlation coefficient $\alpha$ of the questionnaire reliability is 0.909 .

\subsection{Experiment Analysis}




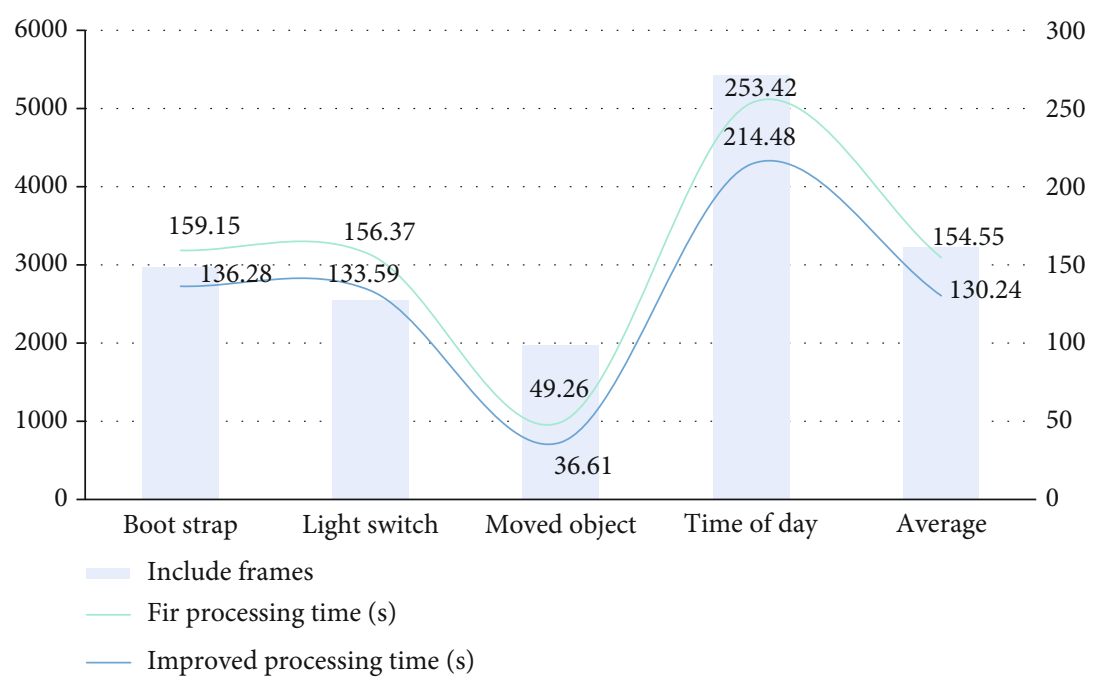

FIGURE 7: Algorithm processing speed comparison.

TABLE 8: Experimental test results.

\begin{tabular}{|c|c|c|c|c|c|}
\hline & Sport mode & Light changes & Number of experiments & Number of alarms & Alarm accuracy \\
\hline 1 & Walk normally & Mutation & 20 & 19 & $95.0 \%$ \\
\hline 2 & Walk normally & Normal & 20 & 20 & $100.0 \%$ \\
\hline 3 & Walk slowly & Normal & 20 & 19 & $95.0 \%$ \\
\hline 4 & Running fast & Normal & 20 & 18 & $90.0 \%$ \\
\hline 5 & Bent over to walk & Normal & 20 & 19 & $95.0 \%$ \\
\hline 6 & Free pose & Normal & 20 & 17 & $85.0 \%$ \\
\hline
\end{tabular}

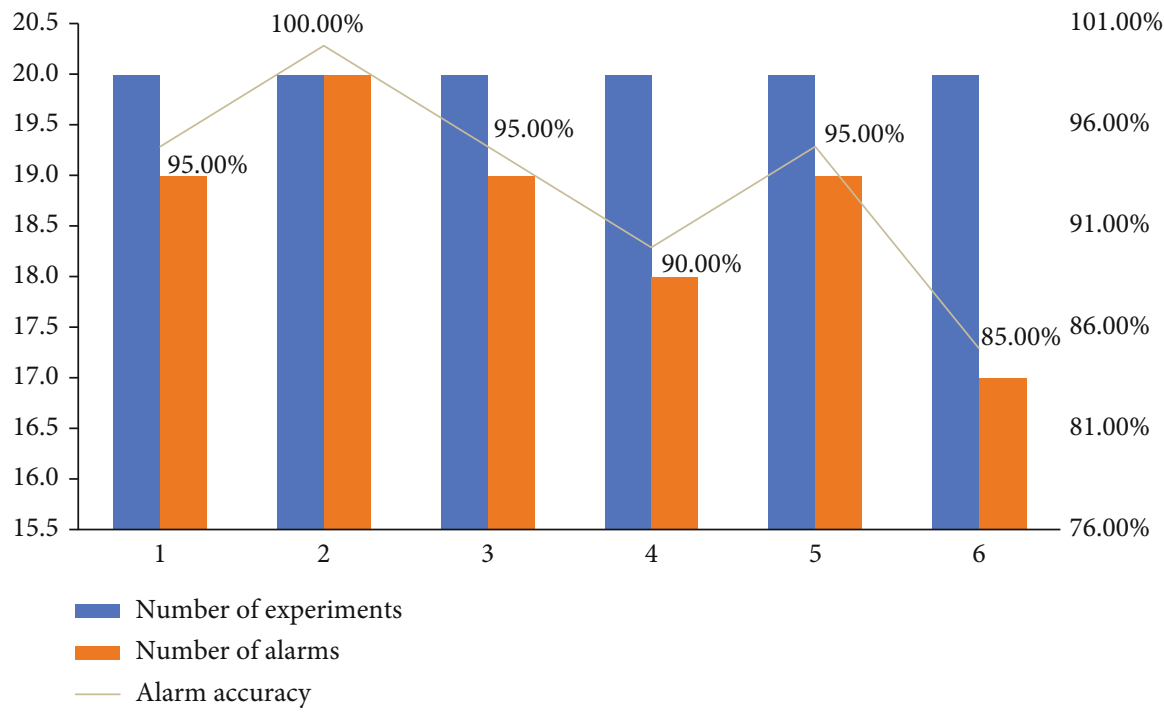

Figure 8: Experimental test results.

(1) Use the original FIR filter processing algorithm and the improved FIR filter processing algorithm to analyze the "Bootstrap," "LightSwitch," "MovedObject," and "Time Of Day" videos in the Wallflower public image database. FIR filters are widely used in digital signal processing, and their main function is to filter out uninteresting signals and leave useful signals. The FIR filter has an all-zero structure, and the 
system is always stable, and it has the characteristic of linear phase, and there is no distortion in the phase of all signals within the effective frequency range. Sequences are tested, and the time required for them to run is shown in Table 7 and Figure 7

It can be seen from the chart that thereby increases the overall complexity of the algorithm, but compared to the original algorithm has a shorter running time, the original FIR processing speed is 20.9 frames per second, and the improved algorithm as a whole reaches a processing speed of 24.8 frames per second.

(2) Under the condition of normal light changes, exercise for normal walking, slow walking, bending walking, running, and free postures within the specified range (free postures include all postures except the several exercise modes described above), children of different genders and body types are monitored, including those who participate in training and those who are not; use fluorescent lamps to simulate sudden changes in light, and monitor children who exercise normally within a specified range. The exercise monitoring results are shown in Table 8 and Figure 8

From the results in the figure, the average monitoring accuracy rate of the monitoring system is $93.33 \%$, which is quite accurate. Under the condition of normal light change, the algorithm has very high alarm accuracy for normal walking and fast running children and children with various sports modes. Experimental data shows that the algorithm can effectively detect slow-moving children, but underreporting will still occur when the children are moving very slowly. When infants bend over and walk, the correct rate of alarm is 95.0\%. The reason is that the infant training samples are mainly for children in various sports. For children with free posture exercise, algorithm's alarm accuracy rate is $85.0 \%$. The main reason for underreporting is the limited number and patterns of children's training samples. For example, there is no sample training for crawling children. Underreporting may occur at the same time. Under the condition of sudden changes in light, the average number of children detected is reduced compared with that under normal light changes.

\section{Conclusions}

The development of computer-related software and hardware is also rapidly developing towards miniaturization, portability, and intelligence. Embedded intelligent monitoring system is one of the hottest research needs. It can be associated with various things in social life to form a research project. For example, the real-time control of the monitoring of school bus ride dynamics can reduce the occurrence of accidents. The ability of the microprocessor to process data quickly and the convenient and easy-to-use network transmission capabilities, including the high reliability, real-time, and low power consumption of embedded systems, can promote its widespread application and development. Its reliability can make its application range wider and ensure the privacy of users. As a universal requirement of embedded systems, real-time performance is an indicator of the customer and designer's emphasis on examination rate; low power consumption can solve the energy equipment of small systems problem; these points are important features of embedded systems welcomed by the majority of users.

The intelligent monitoring system is a multidisciplinary problem. This article focuses on the detection of children's sports targets and the recognition of objects in the background of sports training. Use monitoring technology under the Internet of Things environment to collect data on the actual information of children's physical exercise training. Through the monitoring of the Internet of Things, through video and sound monitoring, the information content can be conveyed intuitively, accurately, and in time, to realize the linkage reaction between things. The research is based on the summary and analysis of existing research results. Due to the limited resources of the platform, a method for monitoring sports physique status with low computational complexity and high real-time performance has been designed and implemented.

This article describes in detail the components of the embedded sports infant physical condition real-time monitoring system from both the software and hardware aspects. Through experimental tests, the results show that the embedded sports infant physical condition real-time monitoring system proposed in this article can effectively monitor in real time. The physical information of young children in the sports training scene is displayed, and it is robust to normal light changes. Robustness refers to the robustness of the system, the key to the survival of the system under abnormal and dangerous conditions, and is an important evaluation index for modern control systems. It can show the robustness of the detection system.

\section{Data Availability}

The data underlying the results presented in the study are available within the manuscript.

\section{Conflicts of Interest}

The authors declare that they have no conflicts of interest.

\section{References}

[1] D. Zeng, L. Gu, S. Guo, Z. Cheng, and S. Yu, "Joint optimization of task scheduling and image placement in fog computing supported software-defined embedded system," IEEE Transactions on Computers, vol. 65, no. 12, pp. 3702-3712, 2016.

[2] J. A. Back, L. P. Tedesco, R. F. Molz, and E. O. B. Nara, “An embedded system approach for energy monitoring and analysis in industrial processes," Energy, vol. 115, Part. 1, pp. 811-819, 2016.

[3] P. B. Huang, "An intelligent neural-fuzzy model for an inprocess surface roughness monitoring system in end milling operations," Journal of Intelligent Manufacturing, vol. 27, no. 3, pp. 689-700, 2016.

[4] D. Dormeshkin, A. Gilep, G. Sergeev, and S. Usanov, "Development of CYB5-fusion monitoring system for efficient periplasmic expression of multimeric proteins in 
_Escherichia coli_," Protein Expression and Purification, vol. 128, no. Complete, pp. 60-66, 2016.

[5] S. H. Lee, V. Mariappan, D. C. Won, J. Shin, and S. Yang, "Design of in-situ self-diagnosable smart controller for integrated algae monitoring system," The International Journal of Advanced Culture Technology, vol. 5, no. 1, pp. 64-69, 2017.

[6] B. Rama and I. V. S. Reddy, "Design of bio-medical embedded system for tremor analysis," International Journal of Electronics Engineering Research, vol. 9, no. 7, pp. 1013-1026, 2017.

[7] J. du, R. Li, Z. Xiao, Z. Tong, and L. Zhang, "Optimization of data allocation on CMP embedded system with data migration," International Journal of Parallel Programming, vol. 45, no. 4, pp. 965-981, 2017.

[8] S. S. J. Imtinungla and H. Bordoloi, "Embedded system based agricultural field monitoring system," International Journal of Electronics Engineering Research, vol. 9, no. 7, pp. 977984, 2017.

[9] S. R. Nalamwar, S. Kalhapure, A. Khatake, S. Gandhi, and K. Jain, "Real time communication using embedded system beyond videoconferencing and towards telepresence," International Journal of Computer Applications, vol. 134, no. 14, pp. 28-31, 2016.

[10] M. Jelii, O. Uljevi, and N. Zeni, "Pulmonary function in prepubescent boys: the influence of passive smoking and sports training," Montenegrin Journal of Sports Science \& Medicine, vol. 6, no. 1, pp. 65-72, 2017.

[11] L. Qi and L. Ying, "Retraction notice: the research on the impact of green beans sports drinks on relieving fatigue in sports training," Open Biomedical Engineering Journal, vol. 10, no. 1, pp. 113-113, 2016.

[12] I. Belenkaya, "Musical training of coaches in aesthetic-oriented sports," Slobožans'kij Naukovo-Sportivnij Visnik, vol. 53, no. 3, pp. 7-11, 2016.

[13] M. R. Bohórquez Gómez-Millán and I. Checa Esquiva, "Psychosocial factors related to the competitive anxiety of sports in training stages," Cultura, Ciencia y Deporte, vol. 12, no. 36, pp. 205-210, 2017.

[14] P. J. Du Toit, A. J. Van Rensburg, D. C. Janse Van Rensburg et al., "Influence of two sports vision training techniques on visual skills performance of university students," African Journal for Physical Health Education, Recreation and Dance, vol. 22, no. 2: 1, pp. 428-444, 2016.

[15] M. O. Vyalova, I. M. Sokolov, A. S. Shmoylova, and Y. G. Schwartz, "Hypertension in people in middle and late adulthood during sports and physical training," Cardiovascular Therapy and Prevention (Russian Federation), vol. 19, no. 1, pp. 5-10, 2020.

[16] M. Chai and B. H. Schlingloff, "System monitoring with a fivevalued LTL," Journal of Multiple-Valued Logic and Soft Computing, vol. 26, no. 1-2, pp. 33-54, 2016.

[17] Z. K. Shamsievich, "The creation of a model of development of sports management in improving the physical training and sports management system in Uzbekistan," International Journal of Psychosocial Rehabilitation, vol. 24, no. 5, pp. 6530-6537, 2020.

[18] G. Chen, Y. Lu, B. Li et al., "Mp-rdma: enabling rdma with multi-path transport in datacenters," IEEE/ACM Transactions on Networking, vol. 27, no. 6, pp. 2308-2323, 2019.
[19] O. Weisman, M. Chetouani, C. Saint-Georges et al., "Dynamics of non-verbal vocalizations and hormones during fatherinfant interaction," IEEE Transactions on Affective Computing, vol. 7, no. 4, pp. 337-345, 2016.

[20] X. Li, H. Liu, W. Wang, Y. Zheng, H. Lv, and Z. Lv, "Big data analysis of the internet of things in the digital twins of smart city based on deep learning," Future Generation Computer Systems, vol. 128, pp. 167-177, 2021.

[21] J. I. Olivares and I. A. Aguilera, "Proposal of new regression formulae for the estimation of age in infant skeletal remains from the metric study of the pars basilaris," International Journal of Legal Medicine, vol. 131, no. 3, pp. 1-8, 2016.

[22] J. Kaur and S. Punia, "Floppy infant syndrome: overview," International Journal of Physiotherapy and Research, vol. 4, no. 3, pp. 1554-1563, 2016.

[23] K. E. Kaeppler, R. C. Stetson, B. C. Lanpher, and C. A. Collura, "Infant male with TARP syndrome: review of clinical features, prognosis, and commonalities with previously reported patients," American Journal of Medical Genetics Part A, vol. 176, no. 12, pp. 2911-2914, 2018.

[24] Y. Li, Y. Zuo, H. Song, and Z. Lv, "Deep learning in security of internet of things," IEEE Internet of Things Journal, vol. 8, pp. 1-1, 2021.

[25] Q. Wang, Y. Li, and X. Liu, "Analysis of feature fatigue EEG signals based on wavelet entropy," International Journal of Pattern Recognition and Artificial Intelligence, vol. 32, no. 8, article 1854023, 2018.

[26] C. Li, H. J. Yang, F. Sun, J. M. Cioffi, and L. Yang, “Adaptive overhearing in two-way multi-antenna relay channels," IEEE Signal Processing Letters, vol. 23, no. 1, pp. 117-120, 2016. 